\title{
Quantum Monte Carlo Calculations of the Energy of the Relativistic Homogeneous Electron Gas
}

\author{
S. D. Kenny, ${ }^{1}$ G. Rajagopal,${ }^{1}$ R. J. Needs, ${ }^{1}$ W.-K. Leung, ${ }^{1}$ M. J. Godfrey, ${ }^{2}$ \\ A. J. Williamson, ${ }^{1}$ and W.M.C. Foulkes ${ }^{3}$ \\ ${ }^{1}$ Cavendish Laboratory, Madingley Road, Cambridge CB3 OHE, United Kingdom \\ ${ }^{2}$ Department of Physics, University of Manchester Institute of Science and Technology, P.O. Box 88, \\ Manchester M60 1QD, United Kingdom \\ ${ }^{3}$ The Blackett Laboratory, Imperial College of Science, Technology and Medicine, Prince Consort Road, \\ London SW7 2BZ, United Kingdom
}

(Received 1 May 1996)

\begin{abstract}
The ground state energy of the unpolarized homogeneous electron gas at zero temperature is calculated within the density range $r_{s}=0.1-10$, incorporating relativistic effects via firstorder perturbation theory. Accurate nonrelativistic wave functions and variational and diffusion quantum Monte Carlo techniques are used to calculate the required expectation values. [S0031-9007(96)00783-1]
\end{abstract}

PACS numbers: 71.10.Ca, 31.30.Jv

The homogeneous electron gas (HEG) serves as an important model system in the fields of astrophysics, plasma physics, and condensed matter physics. The most accurate calculations performed to date on the nonrelativistic HEG have used quantum Monte Carlo (QMC) techniques $[1,2]$. In this Letter we extend these studies to include relativistic effects via first-order perturbation theory, calculating the energy of the unpolarized HEG within the density range $r_{s}=0.1-10$.

Previous calculations of the energy of the relativistic HEG have used methods which are equivalent to the summation of "ring diagrams" [3,4], which is an appropriate approximation at very high densities. For our study we have chosen a different approach; we introduce the relativistic effects via first-order perturbation theory including terms of order $1 / c^{2}$, where $c$ is the speed of light $[5,6]$. As we will demonstrate later, this approach is reliable for the HEG in the density regime that we have studied. We use QMC techniques to evaluate the required expectation values, thereby including the effects of electron correlation which strongly influence relativistic effects in this regime. Moreover, there is a considerable literature on relativistic effects in light atoms which we can use to check the validity of the perturbation theory and the accuracy of our QMC methods. Recently we used QMC methods to perform similar calculations of the relativistic energies of several atoms including the ten-electron neon atom [7], which yielded results of very high accuracy.

Derivations of the perturbation to the nonrelativistic Schrödinger equation correct to order $1 / c^{2}$ for a system of electrons in a static electric field have been given by Bethe and Salpeter [5] and Itoh [6]. After averaging over spins the terms with nonzero expectation values for the unpolarized HEG are, in Hartree atomic units,

$$
\begin{aligned}
\hat{\Delta}= & -\sum_{i} \frac{\nabla_{i}^{4}}{8 c^{2}}+\sum_{i} \frac{\pi}{2 c^{2}} \rho_{0}+\sum_{i<j} \frac{\pi}{c^{2}} \delta\left(\mathbf{r}_{i}-\mathbf{r}_{j}\right) \\
& -\sum_{i<j} \frac{1}{2 c^{2}} \overleftarrow{\nabla}_{i} \cdot\left[\frac{\left(\mathbf{r}_{i}-\mathbf{r}_{j}\right)\left(\mathbf{r}_{i}-\mathbf{r}_{j}\right)}{r_{i j}^{3}}+\frac{1}{r_{i j}}\right] \cdot \vec{\nabla}_{j}
\end{aligned}
$$

where $\rho_{0}$ is the charge density of the uniform background, $\mathbf{r}_{i}$ is the position vector of the $i$ th electron, and $r_{i j}$ is the distance between the $i$ th and $j$ th electrons. The arrows above the $\nabla$ symbols indicate the directions in which the gradient operators act. The first term in Eq. (1) arises from the relativistic variation of mass with velocity. The second term is the Darwin interaction of the electrons with the uniform positive background charge density. The third term is a contact interaction arising from the electron-electron Darwin terms and the interaction of electron spin magnetic moments. The fourth term, which we call the retardation term, is the sum of the orbital contribution to the (unretarded) current-current interaction and the retardation correction to the Coulomb potential. The third and fourth terms form the relativistic correction to the electron-electron interaction known as the Breit interaction.

The expectation value of the operators in Eq. (1), evaluated with the nonrelativistic wave function, gives the relativistic correction to the energy to order $1 / c^{2}$. For atoms of low atomic number the $1 / c^{2}$ correction gives a very accurate estimate of the total relativistic correction. There is, however, a small complication in extending the theory to the HEG, in that the perturbation mixes in excited states of the system. This mixing is unimportant in closed shell atoms because the excitation energies are large, but in an infinite HEG there is no gap to excitation. In the unpolarized gas the configurations that are significantly mixed in are those with particle-hole 
pairs very close to the Fermi surface. Their effect on the relativistic correction is small (of higher order than $1 / c^{2}$ ), and can be neglected in the range of densities studied.

We investigate the validity of the $1 / c^{2}$ perturbation theory for the interactions and for the kinetic energy. At the highest density studied $\left(r_{s}=0.1\right)$ the $1 / c^{2}$ correction to the correlation energy is less than $4 \%$ of the $1 / c^{2}$ correction to the exchange energy, so that at high densities it is a good approximation to consider only the exchange energy, which can be evaluated exactly for the relativistic HEG $[3,4]$. The exchange contribution of order $1 / c^{4}$ is small at $r_{s}=0.1$, being only $1.2 \%$ of the $1 / c^{2}$ contribution, and the two corrections become comparable only at $r_{s}=0.01$. At high densities the kinetic energy is also closely approximated by that of a free-electron gas. When $r_{s}=0.1$ the $1 / c^{2}$ mass-velocity term gives the total relativistic contribution to the kinetic energy of the noninteracting HEG to within $0.8 \%$ of the correct value, and the error rises to only $5 \%$ at $r_{s}=0.04$. Radiative corrections are known to be small for atoms with charge densities $r_{s}>0.1$ [8], and they are expected to be even smaller for the HEG in this regime. We therefore believe that the $1 / c^{2}$ perturbation theory is accurate in the density range we have investigated. At very high densities the perturbation theory is not valid, but in this range the relativistic corrections to the electron-electron interaction are negligible compared with the mass-velocity term, which is well approximated by that of the noninteracting system.

We have calculated the expectation values of the terms in Eq. (1) using the variational and diffusion quantum Monte Carlo (VMC and DMC) techniques applied to face-centered cubic simulation cells with periodic boundary conditions. In a VMC calculation an approximate wave function, $\Phi$, must be supplied and the probability distribution, $\Phi^{2}$, is generated via a random walk procedure. After a period of equilibration the required averages are accumulated by summing $\Phi^{-1} \hat{\Delta} \Phi$ along the random walk. For the wave functions, $\Phi$, we used the form

$$
\Phi=D_{\uparrow} D_{\downarrow} \exp \left[-\sum_{i>j} u_{\sigma_{i}, \sigma_{j}}\left(r_{i j}\right)\right],
$$

where $D_{\uparrow}$ and $D_{\downarrow}$ are Slater determinants of plane waves for the up- and down-spin electrons and $u_{\sigma_{i}, \sigma_{j}}\left(r_{i j}\right)$ is a correlation factor which depends on the relative spins of the two electrons. For the $u$ functions we used spherically symmetric forms which act between an electron and the nearest periodic image of the other electrons, and which were optimized by minimizing the variance of the (nonrelativistic) energy. Correlation factors of this type yield excellent results for the HEG [2,9]. To evaluate the nonrelativistic Coulomb interaction we used the nearest-image technique introduced recently by us [10], which gives smaller finite size effects than the standard technique of Ewald summation.
The expectation value of the mass-velocity term was calculated from the integral of $\left(\nabla^{2} \Phi\right)^{2}$, obtained by integrating $\Phi \nabla^{4} \Phi$ by parts. The expectation value of the contact term was calculated by accumulating the pair correlation function for antiparallel-spin electrons using a procedure similar to that described by Ortiz and Ballone [2]. The retardation operator in Eq. (1) falls off slowly (like $1 / r_{i j}$ ) and one might think that this would lead to significant contributions to the expectation value from electrons that are widely separated. In fact, this is not the case and our work shows that the contributions are negligible beyond a screening length. To evaluate the expectation value of the retardation term we used a nearest-image technique and truncated the interaction at the surface of the Wigner-Seitz cell of the simulation cell.

VMC calculations were performed at the densities $r_{s}=0.1,0.3,1,2,5,10$, for simulation cells containing closed shell configurations of 178 and 338 electrons, and in each case the averages were accumulated over more than $10^{6}$ attempted moves of all the electrons. In the DMC method, within the fixed-node approximation, the joint distribution $\Phi \Psi$ is generated via imaginary time evolution of the nonrelativistic equations, where $\Psi$ is the best (lowest energy) wave function consistent with the nodal surface of $\Phi$ [11]. We used the short-time approximation, with time steps of $\Delta t=$ $0.0001,0.001,0.01,0.02,0.04,0.08$ a.u. for the respective densities $r_{s}=0.1,0.3,1,2,5,10$, which gave acceptance/rejection ratios of greater than $99.7 \%$ in each case. DMC calculations were performed with 178 electrons in the simulation cells, and the average number of configurations in the ensemble was 640. After an equilibration period of $10^{3}$ attempted moves of all the electrons in all the configurations, the averages were accumulated over further $5 \times 10^{3}$ attempted moves.

From the averages over the variational distribution $\Phi^{2}$ and the diffusion distribution $\Phi \Psi$ we use the method of extrapolated estimation [11] to approximate the average over the distribution $\Psi^{2}$ with an error only at order $(\Psi-\Phi)^{2}$. The QMC results were corrected for finite size effects using the difference between the Hartree-Fock (HF) expectation values of Eq. (1) calculated for the finite system and the infinite system. For the infinite system the four terms in Eq. (1) give

$$
\Delta_{\mathrm{HF}}=-\frac{(9 \pi / 4)^{7 / 3}}{42 \pi c^{2} r_{s}^{4}}+\frac{3}{8 c^{2} r_{s}^{3}}+\frac{3}{16 c^{2} r_{s}^{3}}+\frac{9}{16 c^{2} r_{s}^{3}}
$$

per electron [12]. In HF theory the contact term has no size dependence, although this is not the case for VMC and DMC calculations. However, for the system sizes used here the pair correlation function is already well converged [2]. The corrections due to extrapolated estimation and finite size corrections are in any case small and their inclusion does not alter the conclusions of our work. 
TABLE I. Expectation values of the first, third, and fourth terms in Eq. (1) as a function of $r_{s}$, in Hartree per electron. Standard deviations in the last significant figures are given in brackets.

\begin{tabular}{lccc}
\hline \hline$r_{s}$ & Mass-velocity & Contact & Retardation \\
\hline 0.1 & $-3.90(2) \times 10^{-1}$ & $8.8(3) \times 10^{-3}$ & $3.01(4) \times 10^{-2}$ \\
0.3 & $-4.87(1) \times 10^{-3}$ & $3.0(2) \times 10^{-4}$ & $1.13(2) \times 10^{-3}$ \\
1 & $-4.45(2) \times 10^{-5}$ & $3.8(2) \times 10^{-6}$ & $3.13(4) \times 10^{-5}$ \\
2 & $-3.42(2) \times 10^{-6}$ & $2.1(2) \times 10^{-7}$ & $4.42(5) \times 10^{-6}$ \\
5 & $-1.43(1) \times 10^{-7}$ & $4(2) \times 10^{-9}$ & $3.84(8) \times 10^{-7}$ \\
10 & $-1.48(1) \times 10^{-8}$ & $0(1) \times 10^{-10}$ & $6.22(5) \times 10^{-8}$ \\
\hline \hline
\end{tabular}

Our final results for the expectation values of the terms in Eq. (1) are shown in Table I. As expected the massvelocity term dominates at high densities. The HF result for the mass-velocity term is accurate at high densities where electron correlation is unimportant. At low densities (large $r_{s}$ ) electron correlation effects strongly modify all of the relativistic corrections. At $r_{s}=10$ our QMC value for the mass-velocity term is about 3 times the HF result; however, at this density the mass-velocity term is small compared with the Breit interaction, which is dominated by the retardation term. At low densities the contact term is very much smaller than the retardation term, which contrasts strongly with HF theory [Eq. (3)] which predicts that the retardation term is 3 times the contact term, independent of density.

One immediate application of our results is to construct an energy functional for use in relativistic local-densityapproximation (LDA) calculations. The relativistic equivalent of the Hohenberg-Kohn theorem was derived by Rajagopal and Callaway [13], while Rajagopal [14] and MacDonald and Vosko [15] dealt explicitly with local approximations to the relativistic energy functional. We define a relativistic correction to the exchange-correlation (XC) energy of the HEG as the expectation value of Eq. (1) (but without the electron-background term) for the interacting system minus two terms. The first of these terms is the expectation value of the mass-velocity term calculated for a noninteracting HEG of the same density [the first term in Eq. (3)], and the second is the direct part of the electron-electron Darwin term, which is generated by the Dirac-Kohn-Sham equations themselves. Note that the expectation value of the electron-background term is equal to minus that of the direct part of the electron-electron Darwin term because the electronic charge density is equal to minus the background density. We also remark that the contribution to the XC energy from the mass-velocity term is considerably smaller than the contribution from the Breit interaction at all densities investigated.

For convenience we have fitted our XC energies with a polynomial expression which multiplies the HF exchange result. The resulting expression is not expected to be

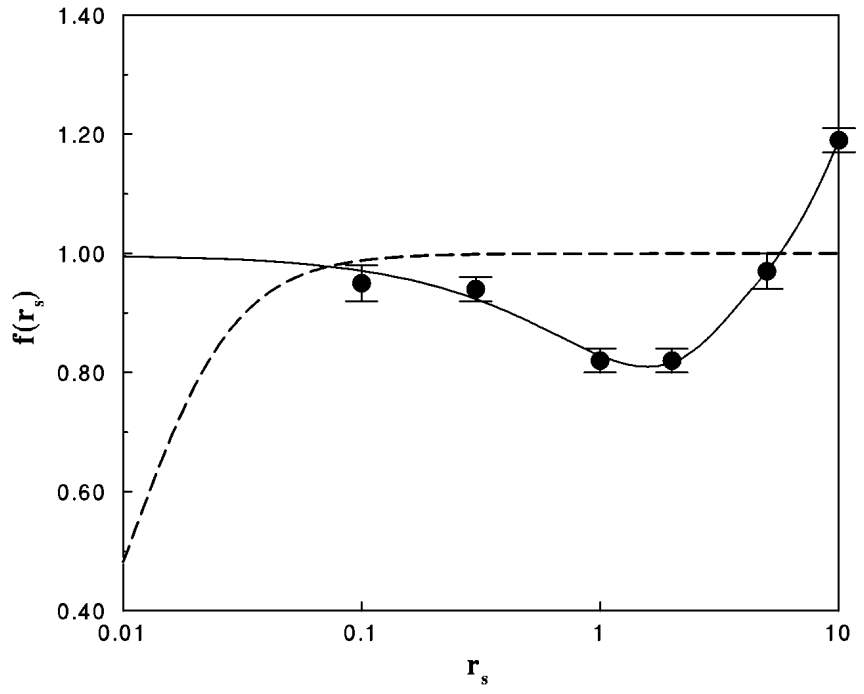

FIG. 1. Relativistic corrections to the exchange-correlation energy of the HEG divided by the HF result $\left(9 / 8 c^{2} r_{s}^{3}\right)$, as a function of the electron density parameter, $r_{s}$. The circles represent our data, while the dashed line is the result quoted by Salpeter [3], which is identical to the exchange part of the expression derived by Jancovici [4]. The dashed line should be accurate for $r_{s}$ well below 0.1, while our QMC data should be accurate at larger $r_{s}$.

reliable far outside the density range $0.1 \leq r_{s} \leq 10$, but at very high densities the $\mathrm{XC}$ correction is negligible compared with the mass-velocity term and at very low densities the entire relativistic correction is negligible. Our XC energy functional is

$$
\begin{aligned}
\epsilon_{\mathrm{xc}}^{\mathrm{rel}} & =\epsilon_{\mathrm{xc}}^{\mathrm{nonrel}}+\frac{9}{8 c^{2} r_{s}^{3}} f\left(r_{s}\right), \\
f\left(r_{s}\right) & = \begin{cases}\sum_{n=0}^{4} c_{n} r_{s}^{n}, & 0 \leq r_{s} \leq 5, \\
a+b r_{s}, & r_{s}>5,\end{cases}
\end{aligned}
$$

where $\epsilon_{\mathrm{xc}}^{\text {nonrel }}$ is the nonrelativistic $\mathrm{XC}$ energy and $c_{0}=0.99818, \quad c_{1}=-0.29020, \quad c_{2}=0.14474, \quad c_{3}=$ $-0.02573, c_{4}=0.001634, a=0.75$, and $b=0.044$. In Fig. 1 we plot our relativistic correction to the $\mathrm{XC}$

TABLE II. Relativistic corrections to the energies of the $\mathrm{He}, \mathrm{Be}^{2+}, \mathrm{Be}, \mathrm{Ne}^{8+}$, and $\mathrm{Ne}$ atoms in Hartree, calculated using two forms of LDA and compared with highly accurate values.

\begin{tabular}{lcrr}
\hline \hline Atom & $\Delta E \mathrm{LDA}^{\mathrm{a}}$ & $\Delta E \mathrm{LDA}^{\mathrm{b}}$ & $\Delta E$ Accurate \\
\hline $\mathrm{He}$ & $+2.5 \times 10^{-5}$ & $+5.0 \times 10^{-5}$ & $-1.04 \times 10^{-4}$ \\
$\mathrm{Be}^{2+}$ & $-8.8 \times 10^{-4}$ & $-7.2 \times 10^{-4}$ & $-2.22 \times 10^{-3}$ \\
$\mathrm{Be}$ & $-9.5 \times 10^{-4}$ & $-7.9 \times 10^{-4}$ & $-2.42 \times 10^{-3}$ \\
$\mathrm{Ne}^{8+}$ & $-8.6 \times 10^{-2}$ & $-8.5 \times 10^{-2}$ & $-1.11 \times 10^{-1}$ \\
$\mathrm{Ne}$ & $-1.04 \times 10^{-1}$ & $-1.03 \times 10^{-1}$ & $-1.37 \times 10^{-1}$ \\
\hline \hline
\end{tabular}

${ }^{a}$ Using our relativistic $\mathrm{XC}$ functional.

${ }^{b}$ Using the relativistic XC functional of Ref. [15]. The accurate values for $\mathrm{He}, \mathrm{Be}^{2+}$, and $\mathrm{Ne}^{8+}$ are from Ref. [16], while those for Be and Ne are from Ref. [7]. 
TABLE III. The contact $(\mathrm{C})$ and retardation $(\mathrm{R})$ terms for the $\mathrm{He}, \mathrm{Be}^{2+}, \mathrm{Be}, \mathrm{Ne}^{8+}$, and $\mathrm{Ne}$ atoms in Hartree, calculated using our new LDA and compared with highly accurate values. The accurate values for $\mathrm{He}, \mathrm{Be}^{2+}$, and $\mathrm{Ne}^{8+}$ are from Ref. [16], while those for Be and Ne are from Ref. [7].

\begin{tabular}{lcccc}
\hline \hline Atom & $\mathrm{C}(\mathrm{LDA})$ & $\mathrm{C}($ Accurate $)$ & $\mathrm{R}(\mathrm{LDA})$ & $\mathrm{R}($ Accurate $)$ \\
\hline $\mathrm{He}$ & $1.65 \times 10^{-5}$ & $1.78 \times 10^{-5}$ & $9.37 \times 10^{-5}$ & $-7.41 \times 10^{-6}$ \\
$\mathrm{Be}^{2+}$ & $2.45 \times 10^{-4}$ & $2.55 \times 10^{-4}$ & $9.78 \times 10^{-4}$ & $-4.68 \times 10^{-5}$ \\
$\mathrm{Be}$ & $2.58 \times 10^{-4}$ & $2.72 \times 10^{-4}$ & $1.03 \times 10^{-3}$ & $-5.18 \times 10^{-5}$ \\
$\mathrm{Ne}^{8+}$ & $5.40 \times 10^{-3}$ & $5.46 \times 10^{-3}$ & $1.80 \times 10^{-2}$ & $-3.73 \times 10^{-4}$ \\
$\mathrm{Ne}$ & $6.26 \times 10^{-3}$ & $6.2 \times 10^{-3}$ & $2.13 \times 10^{-2}$ & $-1.88 \times 10^{-3}$ \\
\hline \hline
\end{tabular}

functional and the MacDonald-Vosko exchange-only expression [15], in both cases divided by the HF result. For $r_{s}>0.1$ the MacDonald-Vosko expression is virtually indistinguishable from the HF result, but our results which include correlation effects differ from the HF result by up to $20 \%$.

We have performed local-density-functional calculations of the ground state total energies of the closed shell $\mathrm{He}, \mathrm{Be}^{2+}, \mathrm{Be}, \mathrm{Ne}^{8+}$, and $\mathrm{Ne}$ atoms. The highest charge density in these atoms corresponds to a local value of $r_{s} \sim 0.1$. Nonrelativistic energies were obtained by self-consistent solution of the single-particle Schrödinger equations of Kohn-Sham density-functional theory, using the standard nonrelativistic XC functional [1]. For the relativistic energies we performed the equivalent calculations using the Dirac equation and relativistic XC functionals. In Table II we report the difference between the relativistic and nonrelativistic atomic energies, calculated with our functional and with the LDA of MacDonaldVosko [15], along with highly accurate values $[7,16]$. The electron-electron effects are most clearly seen in He because for the heavier atoms the mass-velocity and electron-nucleus Darwin terms give the largest contribution to the total relativistic correction. Both our LDA and the MacDonald-Vosko form give the wrong sign for the total relativistic correction in $\mathrm{He}$, and in general the performance of the functionals is not very good, with our functional giving slightly better results in each case. The $1 / c^{2}$ perturbation theory is valid for these atoms $[7,16]$ and therefore we conclude that the major problem arises from the local approximation itself. To investigate this further we have divided the Breit interaction into contact and retardation contributions which are compared with accurate values in Table III. From Table III one can see that the contact term is well described by the LDA but the retardation term is very poorly approximated, with the LDA values being of the wrong order of magnitude and the wrong sign. Indeed by removing the retardation term entirely we can obtain very good values for the Breit interaction in these atoms. However, we would not expect this approach to work in solids where the effects of retardation are expected to be larger and clearly a nonlocal description of the retardation terms is required.
In conclusion, we have calculated the ground state energy of the unpolarized relativistic HEG in the density range $r_{s}=0.1-10$. Relativistic corrections have been included via first-order perturbation theory using QMC methods. We have used our results to investigate the local density approximation for the relativistic exchangecorrelation energy. The contact terms are well described by a local density approximation, but the effects of retardation are very poorly described.

Financial support was provided by EPSRC Grant No. GR/K51198 and EU Contract No. CHRX CT940462. The calculations were performed on the CRAYT3D at EPCC under EPSRC Grant No. GR/K42318.

[1] D. M. Ceperley and B. J. Alder, Phys. Rev. Lett. 45, 566 (1980).

[2] G. Ortiz and P. Ballone, Phys. Rev. B 50, 1391 (1994).

[3] E. E. Salpeter, Astrophys. J. 134, 669 (1961). The relativistic exchange energy quoted in this Letter is credited to the unpublished work of H.S. Zapolski.

[4] B. Jancovici, Nuovo Cimento 25, 428 (1962).

[5] H. A. Bethe and E.E. Salpeter, Quantum Mechanics of One- and Two-Electron Atoms (Springer-Verlag, Berlin, 1957).

[6] T. Itoh, Rev. Mod. Phys. 37, 159 (1965).

[7] S. D. Kenny, G. Rajagopal, and R. J. Needs, Phys. Rev. A 51, 1898 (1995).

[8] G. W.F. Drake, Can. J. Phys. 66, 586 (1988).

[9] A. J. Williamson et al., Phys. Rev. B 53, 9640 (1996).

[10] L. M. Fraser et al., Phys. Rev. B 53, 1814 (1996).

[11] D. M. Ceperley and M. H. Kalos, in Monte Carlo Methods in Statistical Physics, edited by K. Binder (Springer, Berlin, 1979); K. E. Schmidt and M. H. Kalos, in Monte Carlo Methods in Statistical Physics II, edited by K. Binder (Springer, Berlin, 1984).

[12] The expectation values of the contact and retardation terms within HF theory were given earlier by M. Boero and P. Cortona, Phys. Rev. A 49, 825 (1994).

[13] A. K. Rajagopal and J. Callaway, Phys. Rev. B 7, 1912 (1973).

[14] A. K. Rajagopal, J. Phys. C 11, L943 (1978).

[15] A. H. MacDonald and S.H. Vosko, J. Phys. C 12, 2977 (1979).

[16] C. L. Pekeris, Phys. Rev. 112, 1649 (1958). 